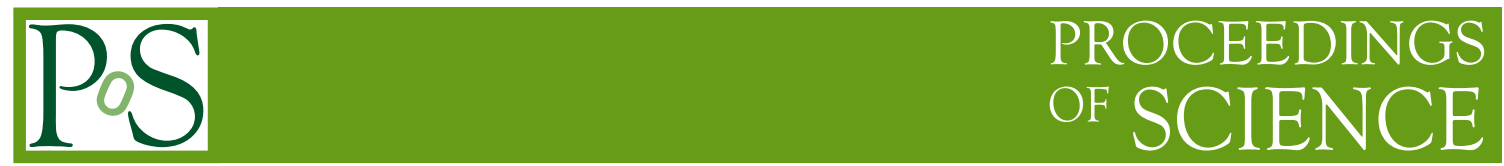

\title{
Integrable models with inverse square potential in maximally symmetric Kähler manifolds
}

\author{
Hovhannes Shmavonyan ${ }^{a, *}$ \\ ${ }^{a}$ A.Alikhanyan National Laboratory, \\ 2 Alikhanian Brothers Street, 0036, Yerevan, Armenia \\ E-mail: hovhannes.shmavonyan@yerphi.am
}

In this paper we consider complex version of models with inverse square potential, namely complex Smorodinsky-Winternitz and complex projective Rosochatius models. These models have interesting properties namely they have hidden symmetries. Due to the complex structure models allow to easily introduce interaction with a constant magnetic field. Also here we provide results for quantization of these models.

RDP online workshop "Recent Advances in Mathematical Physics" - Regio2020,

5-6 December 2020

online

${ }^{*}$ Speaker 


\section{Introduction}

Integrable models are crucial for modern theoretical and mathematical physics. Due to the fact that different physical phenomena can have similar mathematical description, exactly solvable models can be used in many different areas. One can see that when using these models, huge amount of (both macroscopic and microscopic) physical phenomena can be described. Integrable models with inverse square potential have been studied for few decades. Due to this fact they are well studied and there are many important results about these systems. Namely the Calogeromodel has unique properties and due to that nowadays this is an important system in mathematical physics. On the other hand projective spaces have also interesting properties . Due to the fact that they are maximally symmetric spaces it is important to consider physical systems on these spaces. Unfortunately these two branches of mathematical physics are disconnected now. Complex analogs of Calogero model are not studied well and some attempts to construct complexification of Calogerolike models haven't succeeded yet. Possible applications of this work should be highlighted. Namely in condensed matter physics models on complex projective spaces are strongly related with the quantum Hall effect. In High energy physics their role cannot be overestimated. These systems can be viewed as simplified toy models for field theoretical complicated models in high energy research. Our particular example of Calogero model is an example of conformal mechanics. It is well known that conformal symmetry has a crucial role in modern high energy research. In this context supersymmetrization of these systems is also important. Moreover Calogero-like models are strongly related with $A d S_{2} / C F T_{1}$ correspondence [1]. Particularly four-dimensional Hall effect can be related with the systems in $C P^{3}$ [2].

$\mathrm{N}$-dimensional mechanical system (system with $\mathrm{N}$ degrees of freedom) will be called, integrable if it has $\mathrm{N}$ mutually commuting and functionally independent constants of motion[3, 4]. In addition to these constants of motion, the system can have additional ones. In that case we will say that the system is superintegrable. Particularly if $N$-dimensional mechanical system has $2 N-1$ functionally independent constants of motion it will be called maximally superintegrable. In case the system has $\mathrm{N}+1$ conserved quantities it is called minimally superintegrable. While integrable models possess separation of variables in one coordinate system, superintegrability guarantees separation of variables in many coordinate systems. For example two-dimensional oscillator is superintegrable, which allows us to separate variables in Cartesian and polar coordinates. In classical mechanics maximal superintegrability guarantees the closeness of trajectories. Quantum mechanically energy spectrum of integrable models depend on $N$ quantum numbers. If the system has $K$ additional conserved quantities (superintegrable) energy spectrum depends on $N-K$ quantum numbers. For maximal superintegrability we have that the energy spectrum contains only one quantum number. So we can conclude that superintegrability leads to degeneracy of energy spectrum in quantum level.

Smorodinsky-Winternitz model was introduced decades ago [5-7]. After the introduction many articles were devoted to this model [8-15]. Model with inverse square potential on a sphere was introduced by Rosochatius in XIX century. After this the system was rediscovered by other authors $[16,17]$. Many results discussed in this paper are also presented in our previous papers $[18,19]$.

Moreover there was an interest on integrable models on complex and particularly on Kähler 
manifolds. Namely superintegrable oscillator on a complex projective space was introduced and it was shown that inclusion of a constant magnetic field preserves all the symmetries [20,21]. There exists also a generalization of this model on quaternionic spaces [22].

Another important generalization of these models on a Kähler spaces is the supersymmetrization. It was noticed that Kähler structure allows to introduce $N=4$ Supersymmetry, and leads to $S U(2 \mid 1)$ supersymmetry algebra[23-32].

Current paper is a review based on $[18,19]$, and is organized as follows. Firstly there is a brief discussion on complex Euclidean and complex projective spaces, then we present $C^{N}$-Smorodinksy Winternitz model. And finally we discuss $C P^{N}$-Rosochatius system.

\section{2. $C^{N}$ and $C P^{N}$ as Kähler manifolds}

The metric of the N-dimensional complex Euclidean space is well known.

$$
d s^{2}=d z d \bar{z}, \quad g_{a \bar{b}}=\delta_{a \bar{b}} .
$$

It is easy to note the Kähler potential and the symplectic structure is as follows

$$
K(z, \bar{z})=z \bar{z}, \quad \omega=-i d z \wedge d \bar{z}, \quad\left\{z^{a}, \bar{z}^{b}\right\}_{0}=i \delta^{a \bar{b}}
$$

will lead to this well known metric. All the components of Christoffel symbols and Riemann tensor vanish. Finally we present the results for Killing potentials and corresponding Killing vector fields.

$$
\begin{gathered}
h_{a \bar{b}}=\bar{z}^{a} z^{b}, \quad V_{a \bar{b}}=-i\left(z^{b} \partial_{a}+\bar{z}^{a} \partial_{\bar{b}}\right) \\
h_{a}^{+}=\bar{z}^{a}, \quad V_{a}^{-}=-i \partial_{a}, \quad h_{a}^{-}=z^{a}, \quad V_{a}^{+}=-i \partial_{\bar{a}}
\end{gathered}
$$

$V_{a \bar{b}}$ vector fields generate rotations, while $V_{a}^{-}$and $V_{a}^{+}$are the generators of translation. Although $h_{a \bar{b}}, h_{a}^{+}$and $h_{a}^{-}$are not real, one can take real combinations using these functions. The number of real Killing potentials is $N(2 N+1)$, so as is mentioned $C^{N}$ is maximally symmetric space.

The $N$-dimensional complex projective space is a space of complex rays in the $(N+1)$ dimensional complex Euclidian space $\left(C^{N+1}, \sum_{i=0}^{N} d u^{i} d \bar{u}^{i}\right)$, with $u^{i}$ being homogeneous coordinates of the complex projective space. Equivalently, it can be defined as the quotient $S^{2 N+1} / U(1)$, where $S^{2 N+1}$ is the $(2 N+1)$-dimensional sphere embedded in $C^{N+1}$ by the constraint $\sum_{i=1}^{N} u^{i} \bar{u}^{i}=1$. One can solve the latter by introducing locally "inhomogeneous" coordinates $z_{(i)}^{a}$

$$
z_{(i)}^{a}=\frac{u^{a}}{u^{i}}, \quad \text { with } \quad a \neq i, u^{i} \neq 0 .
$$

Hence, the full complex projective space can be covered by $N+1$ charts marked by the indices $i=0, \ldots, N$, with the following transition functions on the intersection of $i$-th and $j$-th charts:

$$
z_{(i)}^{a}=\frac{z_{(j)}^{a}}{z_{(j)}^{i}} .
$$

Let us endow $C^{N+1}$ with the canonical Poisson brackets $\left\{u^{i}, \bar{u}^{j}\right\}=\imath \delta^{i \bar{j}}$, and define, with respect to them, the $u(N+1)$ algebra formed by the generators

$$
h_{i \bar{j}}=\bar{u}^{i} u^{j} .
$$


Reducing the manifold $C^{N+1}$ by the action of the $U(1)$ group with the generator $h_{0}=\sum_{i=0}^{N} u^{i} \bar{u}^{i}$, we arrive at the $S U(N+1)$-invariant Kä̈ler structure defined by the Fubini-Study metrics

$$
g_{a \bar{b}} d z^{a} d \bar{z}^{b}=\frac{\partial^{2} \log (1+z \bar{z})}{\partial z^{a} \partial \bar{z}^{b}} d z^{a} d \bar{z}^{b}=\frac{d z d \bar{z}}{1+z \bar{z}}-\frac{(\bar{z} d z)(z d \bar{z})}{(1+z \bar{z})^{2}}, \quad K=\log (1+z \bar{z}) .
$$

This metrics is obviously invariant under the passing from one chart to another. Hence, we can omit the indices marking charts and assume, without loss of generality, that we are dealing with 0 -th chart, so that the indices $a, b, c$ run from 1 to $N$.

Being Kähler manifold, the complex projective space is equipped with the Poisson brackets $\left\{z^{a}, \bar{z}^{b}\right\}_{0}=\imath g^{a \bar{b}}$, where $g^{a \bar{b}}=(1+z \bar{z})\left(\delta^{a \bar{b}}+z^{a} \bar{z}^{b}\right)$ is the inverse Fubini-Study metrics. The $s u(N+1)$ isometry of $C P^{N}$ is generated by the holomorphic Hamiltonian vector fields defined as the following momentum maps (Killing potentials).

$$
h_{a \bar{b}}=\frac{\bar{z}^{a} z^{b}}{1+z \bar{z}}, \quad h_{a}^{-}=\frac{\bar{z}^{a}}{1+z \bar{z}}, \quad h_{a}^{+}=\frac{z^{a}}{1+z \bar{z}}
$$

Like for the Euclidean case the number of independent Killing vector fields indicates that this space is again maximally superintegrable. Finally we can compute the components of Christoffel symbol and Riemann tensor.

$$
\Gamma_{b c}^{a}=-\frac{\delta_{b}^{a} \bar{z}^{c}+\delta_{c}^{a} \bar{z}^{b}}{1+z \bar{z}}, \quad R_{a \bar{b} c \bar{d}}=g_{a \bar{b}} g_{c \bar{d}}+g_{c \bar{b}} g_{a \bar{d}}
$$

\section{3. $C^{N}$-Smorodinksy-Winternitz model}

We can introduce SW-system which respects the inclusion of constant magnetic field as follows,

$$
\mathcal{H}=\sum_{a} I_{a}, \quad I_{a}=\pi_{a} \bar{\pi}_{a}+\frac{g_{a}^{2}}{z^{a} \bar{z}^{a}}+\omega^{2} z^{a} \bar{z}^{a},
$$

where $z^{a}, \pi_{a}$ are complex have the following non-zero Poisson brackets

$$
\left\{\pi_{a}, z^{b}\right\}=\delta_{a b}, \quad\left\{\bar{\pi}_{a}, \bar{z}^{b}\right\}=\delta_{a b}, \quad\left\{\pi_{a}, \bar{\pi}_{b}\right\}=\imath B \delta_{a b} .
$$

It is easy to notice that the model can be viewed as a sum of $N$ two-dimensional singular oscillators interacting with constant magnetic field perpendicular to the plane. We see that the Hamiltonians of these two dimensional singular oscillators commute and they commute with $C^{N}$-Smorodinksy Winternitz Hamiltonian.

Every two dimensional singular oscillator has $U(1)$ invariance and they are first order constant of motions

$$
L_{a \bar{a}}=\imath\left(\pi_{a} z^{a}-\bar{\pi}_{a} \bar{z}^{a}\right)-B z^{a} \bar{z}^{a}:\left\{L_{a \bar{a}}, \mathcal{H}\right\}=0
$$

$L_{a \bar{b}}$ being generators of $S U(N)$ algebra are not symmetries of our model

$$
L_{a \bar{b}}=l\left(\pi_{a} z^{b}-\bar{\pi}_{b} \bar{z}^{a}\right)-B \bar{z}^{a} z^{b}: \quad\left\{L_{a \bar{b}}, L_{c \bar{d}}\right\}=i \delta_{a \bar{d}} L_{c \bar{b}}-i \delta_{c \bar{b}} L_{c \bar{d}} .
$$

On the other hand they can be used to construct second order symmetry generators for $C^{N}$ Smorodinksy Winternitz model. 


$$
I_{a b}=L_{a \bar{b}} L_{b \bar{a}}+\left(\frac{g_{a}^{2} z^{b} \bar{z}^{b}}{z^{a} \bar{z}^{a}}+\frac{g_{b}^{2} z^{a} \bar{z}^{a}}{z^{b} \bar{z}^{b}}\right), \quad\left\{I_{a b}, \mathcal{H}\right\}=0, \quad a \neq b
$$

It is easy to compute Poisson brackets between $L_{a \bar{a}}$ and other generators

$$
\left\{L_{a \bar{a}}, I_{b}\right\}=\left\{L_{a \bar{a}}, I_{b c}\right\}=\left\{L_{a \bar{a}}, L_{b \bar{b}}\right\}=\left\{I_{a}, I_{b}\right\}=0
$$

We find that $L_{a \bar{a}}$ commute with others. Other Poisson brackets can be also computed, but they are highly non-trivial

$$
\left\{I_{a}, I_{b c}\right\}=\delta_{a b} S_{a c}-\delta_{a c} S_{a b}, \quad\left\{I_{a b}, I_{c d}\right\}=\delta_{b c} T_{a b d}+\delta_{a c} T_{b c d}-\delta_{b d} T_{a c d}-\delta_{a d} T_{a b c},
$$

where

$$
\begin{gathered}
S_{a b}^{2}=4 I_{a b} I_{a} I_{b}-\left(L_{a \bar{a}} I_{b}+L_{b \bar{b}} I_{a}\right)^{2}-4 g_{a}^{2} I_{b}^{2}-4 g_{b}^{2} I_{a}^{2}-4 \omega^{2} I_{a b}\left(I_{a b}-L_{a \bar{a}} L_{b \bar{b}}\right) \\
+4 \omega^{2} g_{b}^{2} L_{a \bar{a}}^{2}+4 g_{a}^{2} \omega^{2} L_{b \bar{b}}^{2}+16 g_{a}^{2} g_{b}^{2} \omega^{2}-2 B\left(I_{a b}-L_{a \bar{a}} L_{b \bar{b}}\right)\left(L_{a \bar{a}} I_{b}+L_{b \bar{b}} I_{a}\right) \\
-B^{2}\left(I_{a b}-L_{a \bar{a}} L_{b \bar{b}}\right)^{2}+4 B\left(g_{b}^{2} I_{a} L_{a \bar{a}}+g_{a}^{2} I_{b} L_{b \bar{b}}\right)+4 B^{2} g_{a}^{2} g_{b}^{2} \\
T_{a b c}^{2}=2\left(I_{a b}-L_{a \bar{a}} L_{b \bar{b}}\right)\left(I_{b c}-L_{b \bar{b}} L_{c \bar{c}}\right)\left(I_{a c}-L_{a \bar{a}} L_{c \bar{c}}\right)+2 I_{a b} I_{a c} I_{b c}+L_{a \bar{a}}^{2} L_{b \bar{b}}^{2} L_{c \bar{c}}^{2} \\
-4\left(g_{c}^{2} I_{a b}\left(I_{a b}-L_{a \bar{a}} L_{b \bar{b}}\right)+g_{a}^{2} I_{b c}\left(I_{b c}-L_{b \bar{b}} L_{c \bar{c}}\right)+g_{b}^{2} I_{a c}\left(I_{a c}-L_{a \bar{a}} L_{c \bar{c}}\right)\right) \\
-\left(I_{b c}^{2} L_{a \bar{a}}^{2}+I_{a b}^{2} L_{c \bar{c}}^{2}+I_{a c}^{2} L_{b \bar{b}}^{2}\right)+4 g_{b}^{2} g_{c}^{2} L_{a \bar{a}}^{2}+4 g_{a}^{2} g_{c}^{2} L_{b \bar{b}}^{2}+4 g_{a}^{2} g_{b}^{2} L_{c \bar{c}}^{2}+16 g_{a}^{2} g_{b}^{2} g_{c}^{2}
\end{gathered}
$$

Symmetry algebra can be modified and can be written in a simpler form, if we introduce the following generators

$$
M_{a a}=L_{a \bar{a}}^{2}+4 g_{a}^{2}, \quad M_{a b}=I_{a b}-\frac{1}{2} L_{a \bar{a}} L_{b \bar{b}}, \quad M_{a 0}=I_{a}-\frac{B}{2} L_{a \bar{a}}, \quad M_{00}=4 \omega^{2}+B^{2} .
$$

Since $L_{a \bar{a}}$ commute with all other generators Poisson brackets of $M$ will exactly coincide with the Poisson brackets of $I_{a b}$ and $I_{a}$.

$$
\left\{M_{a b}, M_{c d}\right\}=\delta_{b c} T_{a b d}+\delta_{a c} T_{b c d}-\delta_{b d} T_{a c d}-\delta_{a d} T_{a b c}, \quad\left\{M_{a 0}, M_{a b}\right\}=\delta_{a b} S_{a c}-\delta_{a c} S_{a b} .
$$

where

$$
\begin{gathered}
S_{a b}^{2}=4 M_{a b} M_{a 0} M_{b 0}+\left(\omega^{2}+\frac{B^{2}}{4}\right)\left(M_{a a} M_{b b}-4 M_{a b}^{2}\right)-M_{b 0}^{2} M_{a a}-M_{a 0}^{2} M_{b b} \\
T_{a b c}^{2}=4 M_{a b} M_{b c} M_{a c}-M_{a b}^{2} M_{c c}-M_{a c}^{2} M_{b b}-M_{b c}^{2} M_{a a}+\frac{1}{4} M_{a a} M_{b b} M_{c c}
\end{gathered}
$$

Moreover we can introduce indices with capital letters, where they take values from 0 to $\mathrm{N}$. Using these notations we have

$$
\left\{M_{A B}, M_{C D}\right\}=\delta_{B C} R_{A B D}+\delta_{A C} R_{B C D}-\delta_{B D} R_{A C D}-\delta_{A D} R_{A B C}
$$

where

$$
R_{A B C}^{2}=4 M_{A B} M_{B C} M_{A C}-M_{A B}^{2} M_{C C}-M_{A C}^{2} M_{B B}-M_{B C}^{2} M_{A A}+\frac{1}{4} M_{A A} M_{B B} M_{C C}
$$


$R_{A B C}$ are antisymmetric, whereas $M_{A B}$ are symmetric.

We can present the result for the quantum solution. To solve the Schrödinger Equation we can use the fact that the system is a sum of two dimensional Hamiltonians and separate the variables. Moreover using diagonal elements of $S U(N)$ generators we can separate variables in each two dimensional problem and obtain one dimensional equations. The final result for the wave functions of two-dimensional system and the energy spectrum are as follows

$$
\begin{aligned}
\psi(z, \bar{z}, n, m)= & \frac{C_{n, m}}{\sqrt{2 \pi}}(\sqrt{z / \bar{z}})^{m} F\left(-n, \sqrt{m^{2}+\frac{4 g^{2}}{\hbar^{2}}}+1, \frac{2 \sqrt{\omega^{2}+\frac{B^{2}}{4}}}{\hbar} z \bar{z}\right) \times \\
& \times\left(\frac{2 \sqrt{\omega^{2}+\frac{B^{2}}{4}}}{\hbar} z \bar{z}\right)^{1 / 2 \sqrt{m^{2}+\frac{4 g^{2}}{\hbar^{2}}}} e^{-\frac{2 \sqrt{\omega^{2}+\frac{B^{2}}{4}}}{\hbar}} z \bar{z} \\
E= & \hbar \sqrt{\omega^{2}+\frac{B^{2}}{4}}\left(2 n+1+\sqrt{m^{2}+\frac{4 g^{2}}{\hbar^{2}}}\right)+\frac{B \hbar m}{2}
\end{aligned}
$$

So the final solution can be found, since the wavefunction is the product of two two dimensional wavefunctions, whereas the energy spectrum is the sum.

$$
\begin{gathered}
\Psi(z, \bar{z})=\prod_{a=1}^{N} \psi\left(z_{a}, \bar{z}_{a}, n_{a}, m_{a}\right) \\
E_{t o t}=\sum_{a=1}^{N} E_{n_{a}, m_{a}}=\hbar \sqrt{\omega^{2}+\frac{B^{2}}{4}}\left(2 n+N+\sum_{a=1}^{N} \sqrt{\left.m_{a}^{2}+\frac{4 g_{a}^{2}}{\hbar^{2}}\right)}+\frac{B \hbar}{2} \sum_{a=1}^{N} m_{a},\right. \\
n=\sum_{a=1}^{N} n_{a}, \quad n=0,1,2 \ldots \quad m_{a}=0, \pm 1, \pm 2, \ldots
\end{gathered}
$$

We can notice that the energy spectrum of the $C^{N}$-Smorodinky-Winternitz system depends on $N+1$ quantum numbers, namely $n$ and $m_{a}$.

We can briefly discuss the number of conserved quantities. We have $N$ real functionally independent constants of motion $\left(I_{a}\right)$. Moreover let us mention that $I_{a b}$ are real, and although it has $N(N-1) / 2$ components, the number of functionally independent constants of motion is $N-1$. In addition to this, the complex system has $N$ real conserved quantities $\left(L_{a \bar{a}}\right)$. So the total number of constants of motion is $3 N-1$ and it is superintegrable (but not maximally superintegrable). Let us remind that the spectrum of $D$-dimensional quantum mechanics with $D+K$ independent integrals of motion depends on $D-K$ quantum numbers Specifically if $N=1$ the system is integrable. For $N=2$ the system is superintegrable, but it has only one additional constant of motion (minimally superintegrable).

\section{4. $C P^{N}$-Rosochatius system}

$C P^{N}$-Rosochatius system" can be defined by Hamiltonian

$$
\mathcal{H}_{\text {Ros }}=g^{a \bar{b}} \pi_{a} \bar{\pi}_{b}+(1+z \bar{z})\left(\omega_{0}^{2}+\sum_{a=1}^{N} \frac{\omega_{a}^{2}}{z^{a} \bar{z}^{a}}\right)-\sum_{i=0}^{N} \omega_{i}^{2},
$$


where $g^{a \bar{b}}=(1+z \bar{z})\left(\delta^{a \bar{b}}+z^{a} \bar{z}^{b}\right)$ is the inverse Fubini-Study metrics.

The symplectic structure is as follows

$$
\left\{\pi_{a}, z^{b}\right\}=\delta_{a}^{b}, \quad\left\{\bar{\pi}_{a}, \bar{z}^{b}\right\}=\delta_{\bar{a}}^{\bar{b}}, \quad\left\{\pi_{a}, \bar{\pi}_{b}\right\}=\imath B g_{\bar{a} b}
$$

The model has $N$ manifest (kinematical) $U(1)$ symmetries with the generators

$$
J_{a \bar{a}}=\imath \pi_{a} z^{a}-\imath \bar{\pi}_{a} \bar{z}^{a}-B h_{a \bar{a}}: \quad\left\{J_{a \bar{a}}, \mathcal{H}\right\}=0,
$$

and hidden symmetries with the second-order generators $I_{i j}=\left(I_{0 a}, I_{a b}\right)$ defined as

$$
I_{0 a}=J_{0 a} \bar{J}_{0 \bar{a}}+\omega_{0}^{2} z^{a} \bar{z}^{a}+\frac{\omega_{a}^{2}}{\bar{z}^{a} z^{a}}, \quad I_{a b}=J_{a \bar{b}} J_{b \bar{a}}+\omega_{a}^{2} \frac{z^{b} \bar{z}^{b}}{z^{a} \bar{z}^{a}}+\omega_{b}^{2} \frac{z^{a} \bar{z}^{a}}{z^{b} \bar{z}^{b}}: \quad\left\{I_{i \bar{j}}, \mathcal{H}\right\}=0 .
$$

Here $J_{a \bar{b}}$ are $S U(N)$ algebra generators. Moreover together with $J_{0 a}$ they form $S U(N+1)$ algebra.

$$
\begin{gathered}
J_{a \bar{b}}=\imath\left(z^{b} \pi_{a}-\bar{\pi}_{b} \bar{z}^{a}\right)-B h_{a \bar{b}}, \quad J_{0 a}=\pi_{a}+\bar{z}^{a}(\bar{z} \bar{\pi})+\imath B h_{a}^{-}: \\
\left\{J_{\bar{a} b}, J_{\bar{c} d}\right\}=i \delta_{\bar{a} d} J_{\bar{b} c}-i \delta_{\bar{c} b} J_{\bar{a} d}, \quad\left\{J_{0 a}, \bar{J}_{b}\right\}=-i\left(J_{a \bar{b}}+\left(\sum_{a=1}^{N} J_{a \bar{a}}+B\right) \delta_{a \bar{b}}\right), \quad\left\{J_{0 a}, J_{b \bar{c}}\right\}=i J_{0 b} \delta_{a \bar{c}}
\end{gathered}
$$

Again we can introduce new indices which take values from 0 to $N$ and they simplify our formulae

$$
I_{i j}=J_{i \bar{j}} J_{j \bar{i}}+\omega_{i}^{2} \frac{u^{j} \bar{u}^{j}}{u^{i} \bar{u}^{i}}+\omega_{j}^{2} \frac{u^{i} \bar{u}^{i}}{u^{j} \bar{u}^{j}} .
$$

In this notation the relevant symmetry algebra is given by the following brackets

$$
\left\{J_{a \bar{a}}, I_{i j}\right\}=0, \quad\left\{I_{i j}, I_{k l}\right\}=\delta_{j k} T_{i j l}+\delta_{i k} T_{j k l}-\delta_{j l} T_{i k l}-\delta_{i l} T_{i j k},
$$

where

$$
\begin{gathered}
\left(T_{i j k}\right)^{2}=2\left(I_{i j}-J_{i \bar{i}} J_{j \bar{j}}\right)\left(I_{j k}-J_{j \bar{j}} J_{k \bar{k}}\right)\left(I_{i k}-J_{i \bar{i}} J_{k \bar{k}}\right)+2 I_{i j} I_{i k} I_{j k}+J_{i \bar{i}}^{2} J_{j \bar{j}}^{2} J_{k \bar{k}}^{2} \\
-4\left(\omega_{k}^{2} I_{i j}\left(I_{i j}-J_{i \bar{i}} J_{j \bar{j}}\right)+\omega_{i}^{2} I_{j k}\left(I_{j k}-J_{j \bar{j}} J_{k \bar{k}}\right)+\omega_{j}^{2} I_{i k}\left(I_{i k}-J_{i \bar{i}} J_{k \bar{k}}\right)\right) \\
+4 \omega_{j}^{2} \omega_{k}^{2} J_{i \bar{i}}^{2}+4 \omega_{i}^{2} \omega_{k}^{2} J_{j \bar{j}}^{2}+4 \omega_{i}^{2} \omega_{j}^{2} J_{k \bar{k}}^{2}+16 \omega_{i}^{2} \omega_{j}^{2} \omega_{k}^{2}-\left(I_{j k}^{2} J_{i \bar{i}}^{2}+I_{i j}^{2} J_{k \bar{k}}^{2}+I_{i k}^{2} J_{j \bar{j}}^{2}\right)
\end{gathered}
$$

We also provide the energy spectrum of the quantized model

$$
\begin{gathered}
\left.E_{n,\left\{m_{a}\right\}=\frac{\hbar^{2}}{4}(2 n+N}+\sqrt{\left(B / \hbar+\sum_{a=1}^{N} m_{a}\right)^{2}+4 \omega_{0}^{2}}+\sum_{a=1}^{N} \sqrt{4 \omega_{a}^{2}+m_{a}^{2}}\right)^{2}- \\
-\frac{B^{2}+\hbar^{2} N^{2}}{4}-\hbar^{2} \sum_{i=0}^{N} \omega_{i}^{2},
\end{gathered}
$$

where $n=\sum_{a=1}^{N} n_{a}=0,1, \ldots$ and $m_{a}=0, \pm 1, \pm 2, \ldots$

Thus the spectrum of quantum $C P^{N}$-Rosochatius system depends on $N+1$ quantum numbers. This is in full agreement with the fact that this system has $3 N-1$ functionally independent constants of motion. So we have the same number of functionally independent constants of motion as for the $C^{N}$-Smorodinksy-Winternitz model. 


\section{Acknowledgments}

As was mentioned the paper is a review on a work done in collaboration with professor Armen Nersessian and Evgeny Ivanov. The author acknowledges professor Armen Nersessian for support, courage and for crucial discussions. Moreover I would like to acknowledge professor Evgeny Ivanov and Stepan Sidorov for contribution and valuable support.

The author also acknowledges a partial financial support of Armenian Science Committee (grant 20RF-023), and of the Regional Doctoral Program on Theoretical and Experimental Particle Physics Program sponsored by VolkswagenStiftung. This work was fulfilled within the ICTP Affiliated Center Program AF-04 and ICTP Network project NT-04.

\section{References}

[1] C. Chamon, R. Jackiw, S.-Y. Pi, L. Santos Conformal quantum mechanics as the CFT1 dual to AdS2 MIT-CTP/4270 arXiv:1106.0726

[2] S. Bellucci, P. Casteill and A. Nersessian Four-dimensional Hall mechanics as a particle on $C P^{3}$ Phys.Lett. B574 (2003) 121-128 arXiv:hep-th/0306277

[3] V.I. Arnold Mathematical methods of classical mechanics, "Nauka" Publ., Moscow, 1989

[4] A. M. Perelomov, Integrable systems of classical mechanics and Lie algebras, Birkhauser, 1990.

[5] I. Fris, V. Mandrosov, Ya. A. Smorodinsky, M. Uhlir, and P. Winternitz, On higher symmetries in quantum mechanics, Phys. Lett. 16 (1965) 354;

[6] P. Winternitz, Ya. A. Smorodinsky, M. Uhlir, I. Fris, Symmetry groups in classical and quantum mechanics, Soviet J. Nuclear Phys. 4 (1967), 444;

[7] A. A. Makarov, Ya. A. Smorodinsky, Kh. Valiev, and P. Winternitz, A systematic search for non-relativistic system with dynamical symmetries, Nuovo Cim. A 52 (1967) 1061.

[8] N. W. Evans Super-integrability of the Winternitz system Phys. Lett. A, 147(1990)483-486

[9] N. W. Evans, Superintegrability in classical mechanics,Phys. Rev. A 41(1990) 5666-5676;

[10] N. W. Evans, Group theory of the Smorodinsky-Winternitz system,J. Math. Phys. 32(1991) 3369-3375

[11] Heinzl, T., Ilderton A. Superintegrable relativistic systems in spacetime-dependent background fields. Journal of Physics A: Mathematical and Theoretical 50 (2017) 345204

[12] E. G. Kalnins, G. C. Williams, W. Miller Jr. and G. S. Pogosyan, Superintegrability in threedimensional Euclidean space, J. Math.Phys. 40(1999)708-725

[13] M. F. Hoque, I. Marquette and Y. Z. Zhang, Recurrence approach and higher rank cubic algebras for the $N$-dimensional superintegrable systems, J. Phys. A 49 (2016) no.12, 125201 [arXiv:1511.03331 [math-ph]]; 
[14] M. F. Hoque, I. Marquette and Y. Z. Zhang, Quadratic algebra structure and spectrum of a new superintegrable system in N-dimension, J. Phys. A 48 (2015) no.18, 185201.

[15] W. Miller Jr, S. Post, P. Winternitz Classical and quantum superintegrability with applications. Journal of Physics A: Mathematical and Theoretical, 46(2013) 423001

[16] J. Harnad and O. Yermolayeva, Superintegrability, Lax matrices and separation of variables, CRM Proc. Lect. Notes 37 (2004) 65 [nlin/0303009 [nlin.SI]].

[17] A. Galajinsky, A. Nersessian, and A. Saghatelian, Superintegrable models related to near horizon extremal Myers-Perry black hole in arbitrary dimension, JHEP 2013 (2013) 2,

[18] H. Shmavonyan $C^{N}$-Smorodinsky-Winternitz system in a constant magnetic field Physics Letters A 383 (12) 1223-1228 (2019)

[19] E. Ivanov, A. Nersessian, H. Shmavonyan $C P^{N}$-Rosochatius system, superintegrability, supersymmetry Phys. Rev. D 99, 085007 (2019) arXiv:1812.00930

[20] S. Bellucci and A. Nersessian, (Super)oscillator on $C P^{N}$ and constant magnetic field, Phys. Rev. D 67 (2003) 065013 [hep-th/0211070];

[21] S. Bellucci, A. Nersessian and A. Yeranyan Quantum oscillator on $C P^{N}$ in a constant magnetic field Phys.Rev. D70 (2004) 085013 [hep-th/0406184]

[22] S. Bellucci, S. Krivonos, A. Nersessian and V. Yeghikyan, Isospin particle systems on quaternionic projective spaces, Phys. Rev. D 87 (2013) no.4, 045005 [arXiv:1212.1663 [hep-th]].

[23] E. Ivanov and S. Sidorov, Deformed Supersymmetric Mechanics, Class. Quant. Grav. 31 (2014) 075013, [arXiv:1307.7690 [hep-th]];

[24] E. Ivanov and S. Sidorov, Super Kähler oscillator from SU(2|1) superspace, J. Phys. A 47 (2014) 292002, [arXiv:1312.6821[hep-th]];

[25] E. Ivanov, S. Sidorov and F. Toppan, Superconformal mechanics in SU(2|1) superspace, Phys. Rev. D 91(2015)085032,[arXiv:1501.05622[hep-th]];

[26] E. Ivanov, O. Lechtenfeld and S. Sidorov, SU(2|2) supersymmetric mechanics, JHEP 1611(2016) 031,[arXiv:1609.00490[hep-th]];

[27] N. Kozyrev, S. Krivonos, O. Lechtenfeld and A. Sutulin, $S U(2 \mid 1)$ supersymmetric mechanics on curved spaces, JHEP 1805 (2018) 175, [arXiv:1712.09898 [hep-th]];

[28] E. Ivanov, O. Lechtenfeld and S. Sidorov, Deformed $N=8$ mechanics of $(8,8,0)$ multiplets JHEP 1808 (2018) 193, [arXiv:1807.11804[hep-th]].

[29] S. Bellucci and A. Nersessian, A Note on $N=4$ supersymmetric mechanics on Kahler manifolds, Phys. Rev. D 64 (2001) 021702, [hep-th/0101065]. 
[30] S. Bellucci and A. Nersessian, Supersymmetric Kahler oscillator in a constant magnetic field, Proc. of 5th Int. Workshop on Supersymmetries and Quantum Symmetries, Dubna, July 24 29, 2003, Ed. E.Ivanov and A. Pashnev, [hep-th/0401232].

[31] E. Ivanov, A. Nersessian, S. Sidorov, H. Shmavonyan Symmetries of deformed supersymmetric mechanics on Kähler manifolds Phys.Rev.D 101 (2020) 2, 025003

[32] E. Ivanov, A. Nersessian, S. Sidorov Quantum $S U(2 \mid 1)$ supersymmetric $C^{N}$ SmorodinskyWinternitz system JHEP 01 (2021) 015 